\title{
A COMPARISON OF L-BAND AND C-BAND RF GUNS AS SOURCES FOR INLINE-INJECTION SYSTEMS
}

\author{
Juan C. Gallardo and Harold G. Kirk \\ Physics Department, Bldg. 901-A \\ Brookhaven National Laboratory \\ Upton, New York 11973-5000 \\ and \\ Thomas Meyerer \\ Fachochscule Ostfriesland \\ Constantiaplatz 4 \\ D-2970 Emdem, Germany
}

\begin{abstract}
We consider the beam dynamics associated with installing a BNL type $1 \frac{1}{2}$ cell L-band or C-band if gun before two TESLA L-band cryomodules. This system will deliver a 25 $\mathrm{MeV}$ electron beam with peak currents on the order of 100 A suitable for further magnetic compression. We evaluate the injection systems utilizing the electron beam dynamic code PARMELA from the point of view of minimizing the transverse invariant emittance.
\end{abstract}

\section{INTRODUCTION}

High-brightness beams are of great interest to the accelerator physics community because of the promise they hold to access new regimes of physics. These regimes include high-luminosity colliders, high-gain free-electron lasers, and high-brightness photon beams which have applications in both the physical and life sciences. Rf guns remain the focus of efforts to produce such high-brightness beams. L-band (1300 MHz) rf guns built at Los Alamos National Laboratory[1] and S-band (2856 MHz) rf guns built at Brookhaven National Laboratory[2] have demonstrated experimentally the value of this approach.

The dynamics of electron beams produced by $\mathrm{rf}$ guns has been the subject of much attention, especially the beam dynamics pertaining to the production and subsequent dilution of beam emittance in both transverse and longitudinal phase space. The two most significant contributors to emittance dilution in the production of electron beams are the rf dynamics (important for long bunches relative to the period of the rf cycle) within the rf gun and space-charge effects (important for short bunches with high charge content) associated with self fields within the electron bunch. In this paper we will model beams which fall into the later category.

It has been shown that the emittance dilution associated with space-charge effects within an electron bunch can be compensated and reversed by the simple technique of focusing the beam with a solenoidal lens and allowing the beam to drift[3]. If one takes the additional step of accelerating the beam at an appropriate point, it is possible to lock in the reduced emittance of the compensated beam near the minimum value. Further, it has been demonstrated that this technique can be extended beyond the L-band regime to an S-band system[4] which in general has higher accelerating gradients within the if gun.

In this paper we continue to explore this emittance compensation technique by applying it to an of gun-focusing solenoid-superconducting linac system (see Fig. 1). We examine two possible rf guns: a $1 \frac{1}{2}$ cell BNL type 1300 $\mathrm{MHz}$ if gun and a $5200 \mathrm{MHz}$ if gun. Table 1 gives design parameters for the two if guns. For the linac section we model two TESLA 9-cell cryomodules which, when run at accelerating gradients of $10 \mathrm{MV} / \mathrm{m}$, are capable of boosting the beam energy by $20 \mathrm{MeV}$. An important advantage to using superconducting linac sections, aside from the high duty factor, is that the larger apertures of the accelerating structures greatly reduce possible emittance growth due to wake field effects acting on the beam. This has been shown to be potentially harmful to the electron beam when an Sband traveling wave linac is used during the acceleration stage[5].

Table 1. Parameters of the modeled rf guns

\begin{tabular}{lcc}
\hline RF frequency $[\mathrm{MHz}]$ & 1300 & 5200 \\
RF Peak Power $[\mathrm{MW}]$ & 4.8 & 7.7 \\
Field on cathode $[\mathrm{MV} / \mathrm{m}]$ & 50 & 180 \\
Radius of aperture $[\mathrm{cm}]$ & 2.2 & .55 \\
Radius of nose $[\mathrm{cm}]$ & 2.2 & .55 \\
First cell length $[\mathrm{cm}]$ & 5.765 & 1.45 \\
Second cell length $[\mathrm{cm}]$ & 13.53 & 2.90 \\
Gun Exit Energy, MeV & 4.8 & 4.6 \\
\hline
\end{tabular}

\section{BEAM DYNAMICS}

A bright beam at the exit of an rf gun does not assure an equivalent beam at the final focus. The transport of the beam in conjunction with space-charge forces must be an integral part of the study. It has been pointed out in the previous section, that the interplay of space-charge forces with solenoidal focusing forces can lead to a reduction of 


\section{RF Gun with Two TESLA Cryomodules}

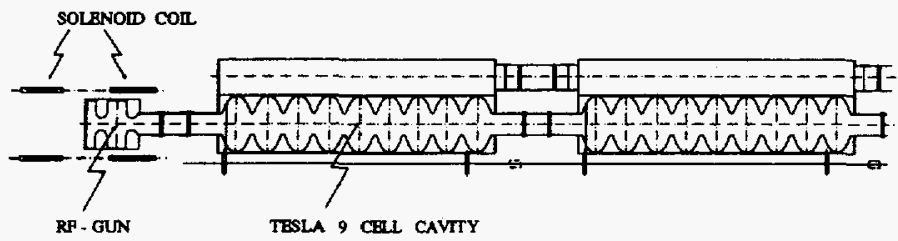

Figure. 1. Schematic showing a general layout of an rf gun-focusing solenoid- superconducting linac system.

the transverse emittance inside the linac. Details of the dynamics of this emittance compensation have already been given in references 4 and 5 .

Our studies have been carried out by modeling the injection system with the $2 \frac{1}{2}$-d simulation code PARMELA[6]. The optimization procedure incorporates the four principle elements of the system: an rf-photocathode gun, a solenoid pair, drift sections, and the linac sections.

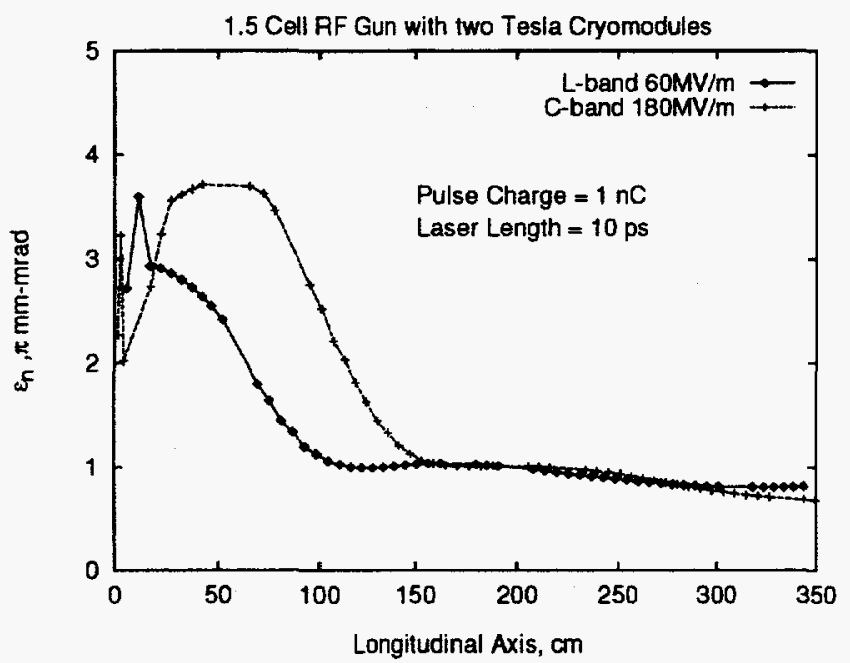

Figure. 2. Invariant transverse emittance $\epsilon_{n}$ vs. the distance of the electron bunch from the cathode for the cases of an L-band (1300 MHz) rf gun and a C-band (5200 MHz) rf gun.

The parameter space for this system is complex. We have chosen to keep some variables fixed while we vary others. We vary the radius and bunch length of the initial electron beam as well as the time of launch relative to the If phase. The bunch charge is fixed at $1 \mathrm{nC}$, the length of the solenoid is fixed at $15 \mathrm{~cm}$, and the average accelerating gradient of the linac sections is set at $10 \mathrm{MV} / \mathrm{m}$. The distance from the cathode to the linac entrance is varied as is the position of the focusing solenoid relative to the cathode. In general, it is found that the solenoid needs to be closer to the cathode when the accelerating gradient within the rf gun is low. A uniform beam distribution is used as it offers significant advantages over Gaussian distributions in terms of minimizing the final beam emittance. Parameters ranges used in this analysis are shown in table 2.

Table 2. Parameters used in the PARMELA simulations for axially symmetric $1 \frac{1}{2}$-cell guns in a $\pi$-mode configuration with a solenoid pair.

\begin{tabular}{|c|c|}
\hline Field on cathode $[\mathrm{MV} / \mathrm{m}]$ & 40 to 300 \\
\hline Initial phase [degree] & 20 to 70 \\
\hline Laser spot size ${ }^{\mathfrak{a}}[\mathrm{cm}]$ & 0.05 to 0.4 \\
\hline Laser pulse width ${ }^{a}[\mathrm{ps}]$ & 5 to 20 \\
\hline Charge $[n C]$ & 1 \\
\hline Solenoidal field $B_{0}[k G]$ & $1.5-5.0$ \\
\hline Solenoid length [cm] & 15.0 \\
\hline
\end{tabular}

${ }^{a}$ Uniform profile

We show in Fig. 2 solutions for delivering a $1 \mathrm{nC}$ beam through the linac. The L-band rf gun result assumes a peak axial electric field of $60 \mathrm{MV} / \mathrm{m}$. It is of particular interest that the emittance compensation technique continues to work for the C-band rf gun operating with peak axial electric fields at $180 \mathrm{MV} / \mathrm{m}$.

We explore this effect further by obtaining the minimum transverse emittances achievable when operating the rf gun with different accelerating gradients. The results of this analysis are shown in Fig. 3. Both rf guns exhibit a strong upturn of emittance at lower accelerating gradients showing that it is advantageous to apply high accelerating gradients at the electron source. These high gradients reduce the development of emittance growth but do not seriously detract from the ability to compensate for the emittance growth that does occur.

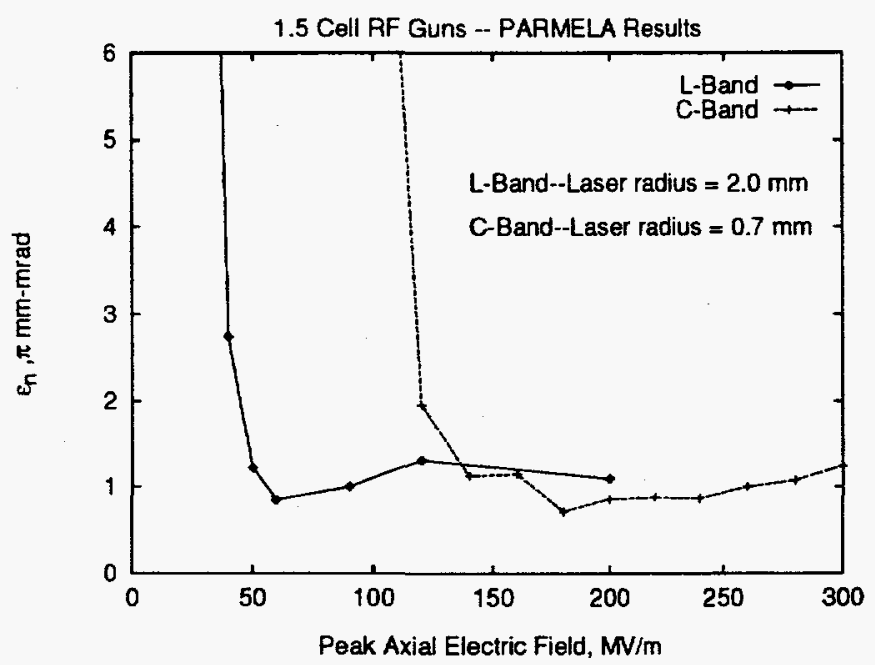

Figure. 3. Invariant transverse emittance $\epsilon_{n}$ vs. the cathode peak electric field for the cases of an L-band (1300 $\mathrm{MHz})$ and a C-band ( $5200 \mathrm{MHz})$ rf gun

\section{CONCLUSIONS}

We have verified that the emittance growth resulting from space-charge induced phase-space dilution can be 
compensated when an L-band rf gun is incorporated into an inline-injection system. In addition, we have shown that this compensation works as well or better when higher accelerating gradients are introduced into the rf gun. Unfortunately, the application of high gradients in an L-band gun is limited by Kilpatrick scaling[7] to axial accelerating fields on the order of $60 \mathrm{MV} / \mathrm{m}$. However, we have shown that it is still possible to achieve good emittance compensation at higher frequencies where higher accelerating gradients are attainable.

\section{ACKNOWLEDGMENTS}

This research was supported by the U.S. Department of Energy under Contract No. DE-ACO2-76-CH00016.

\section{References}

[1] J.S. Fraser et al, Photocathodes in Accelerator Applications, Proceedings of the 1987 IEEE Particle Accelerator Conference, Washington D.C., E. Lindstrom and L.S. Taylor eds. (1987) p. 1705.

[2] K. Batchelor, H. Kirk, J. Sheehan, M. Woodle, K. McDonald, Development of a High-brightness Electron Gun for the Accelerator Test Facility at Brookhaven National Laboratory, Proceedings of the 1988 European Particle Accelerator Conference, Rome, Italy, (1988).

[3] B. Carlsten, New photoelectric injector design for the Los Alamos National Laboratory XUV Fel accelerator, Nucl. Instr. and Meth. A285 (1989) 313.

[4] J. Gallardo and H. Kirk, $A n$ injection scheme for the Brookhaven ATF utilizing space-charge emittance growth compensation, Proceedings of the 1993 Particle Accelerator Conference (1993) 3615.

[5] J. Gallardo and H. Kirk, Optimization of the Brookhaven ATF Inline-injection System Utilizing PARMELA, Submitted to the Proceedings of the 6th Workshop on Advanced Accelerator Concepts, Lake Geneva, Wisconsin, July 12-18, 1994.

[6] L. Young, private communication.

[7] W.D. Kilpatrick, Criterion for Sparking Designed to Include both $R F$ and $D C$, Review of Scientific Instruments, 28, p. 824, 1957. 


\section{DISCLAMIER}

Portions of this document may be illegible in electronic image products. Images are produced from the best available original document. 